\title{
A High-Performance FIR filter Architecture for Reconfigurable Applications
}

\author{
R. Yerriswamy ${ }^{1}$, Dr. D. Vishnu Vardhan ${ }^{2}$, Sankar Lal Sharma ${ }^{3}$ \\ 1,2Department of ECE, JNTUA, Andhra Pradesh, India.
}

${ }^{3}$ Head, Department of ECE, University College of Engineering and Technology, BIKANER, Rajasthan, India

\begin{abstract}
Transpose form finite-impulse response (FIR) filters are characteristically pipelined and support multiple constant multiplications (MCM) procedure that results in significant saving of calculation. However, transpose form configuration does not specifically support the block performing not like direct-form configuration. In this paper, we investigate the possibility of realization of block FIR filter in transpose shape configuration for areadelay efficient realization of huge order FIR filters for both fixed applications. Based on a detailed computational investigation of transpose form configuration of FIR filter, we have derived a flow diagram for transpose shape block FIR filter with reduced register complexity. A detailed block formulation is detailed for transpose form FIR filter. We have inferred a general multiplier-based architecture for the proposed transpose form block filter for reconfigurable applications. A reduced-complex design using multiple constant multiplications scheme is also showed for block implementation of fixed FIR filters. The proposed architecture obtains less area, less delay and less power consumption compared with the existing architecture of direct form structure for medium or long filter lengths. For this project analysis for determining area, power and delay it uses Xilinx.
\end{abstract}

Keywords : Finite-Impulse Response (FIR), Block Processing, Reconfigurable Architecture, Multiple Constant Multiplications (MCM).

\section{INTRODUCTION}

Finite-impulse response filter is mostly used in several digital signal processing (DSP) applications like, loud speaker equalization, echo cancellation, adaptive noise cancellation, speech processing and different communication applications like softwaredefined radio (SDR). Huge numbers of these applications require FIR filters of extensive request to meet the recurrence specifications [2] - [4]. These filters need to help high inspecting rate for rapid computerized correspondence [5]. The number of multiplications and additions required for each filter output notwithstanding, increments straight with the filter arrange.

Since there is no repetitive calculation accessible in the FIR filter algorithm, real-time execution of a large order FIR filter in a resource constrained environment is a challenging task. Filter coefficients frequently stay steady and known apiarian signal processing applications. This feature has been used to diminish the unpredictable for the realization of multiplications. A few designs have suggested by various researchers for efficient realization of FIR filters (having fixed coefficients) utilising distributed 
arithmetic (DA) [18] and multiple constant multiplication (MCM) methods [7], [11]-[13].DAbased plans utilises lookup tables to store recomputed results to decrease the calculations complexity. The MCM technique then again decreases the number of additions essential for the implementations of multiplications by common sub expression sharing, when a given input is multiplied with a group of constants. The MCM technique is most effective, when the common operand was multiplied by more Number of constants. In this way, the MCM scheme is suitable for the realization of large order finite impulse response filters with fixed coefficients. In any case, MCM blocks can be formed only in the transpose form configuration of FIR filters.

Block-processing method was widely used to infer high-throughput hardware structures. It not only gives throughput-scalable design yet additionally enhances the area-delay efficiency. The inference of block-based FIR structure is very clear when directform configuration is utilised [16], though the transpose form configuration does not directly support block preparing. Be that as it may, to take the calculations advantage of the MCM, FIR filter is required to be figured it out by transpose form configuration. Aside from that, transpose form structure is innately pipelined and expected to offer higher working recurrence to support higher sampling rate

\section{IMPLEMENTATION}

To investigate the likelihood for implementing block FIR filter in transpose form configuration with a specific goal to exploit the MCM schemes and the inherent pipelining of area-delay efficient implementation of the large order Finite impulse response filters for fixed applications.

The main contributions are given below.

1. Computational examination of transpose form analysis of finite impulse response filter and dedication of flow chart for transpose form block finite impulse response filter with decreased register complications.

2. Block formulation development for transpose form FIR fixed filter.

3. A reduced-complexity analysis method utilising MCM scheme for block development of fixed finite impulse response filters.

\section{EXISTING SYSTEM}

\section{Computational Analysis}

Suppose in every cycle, the block FIR filter takes a block of $L$ new input samples, and processes those to produce a block of L output samples. The $k^{\text {th }}$ block of filter output $y_{k}$ is computed using the relation $y_{k}=X_{k} \cdot h$

The computation can be expressed in a recurrence form as

$\mathrm{Y}(\mathrm{Z})=S^{0}(\mathrm{z})\left[z^{-1}\left(\ldots\left(z^{-1}\left(z^{-1} c_{M-1}+c_{M-2}\right)+c_{M-3}\right)+\right.\right.$ $\left.\left.\cdots)+c_{1}\right)+c_{0}\right]$

The block transpose form configuration can be derived using this recurrence relation. The delay operator $Z^{-1}$ represents a delay for a block of data in transpose form structure that stores the product of $S_{k}^{0}$ and $c_{m}$.

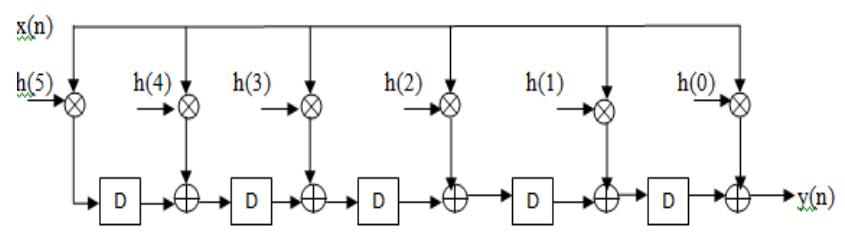

Figure 1. DFG of transpose form structure for output $y(n)$ of length $n=4$

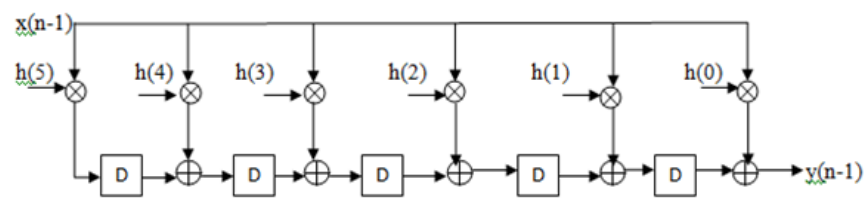

Figure 2. DFG of transpose form structure for output

$$
\mathrm{y}(\mathrm{n}-1) \text { of length } \mathrm{n}=4
$$




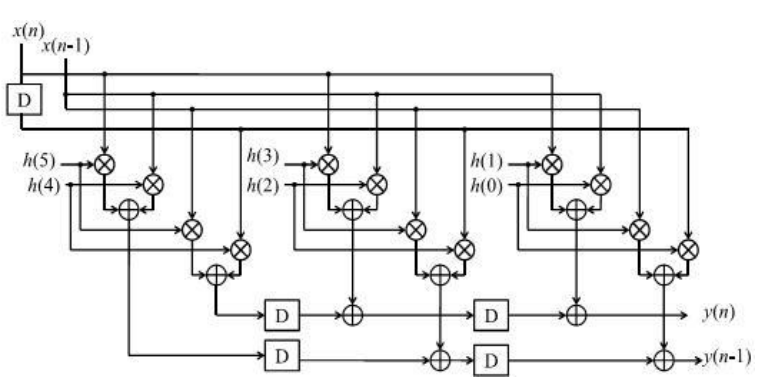

Figure 3. Block Formulation for Transpose Form FIR Filter

\section{PROPOSED SYSTEM}

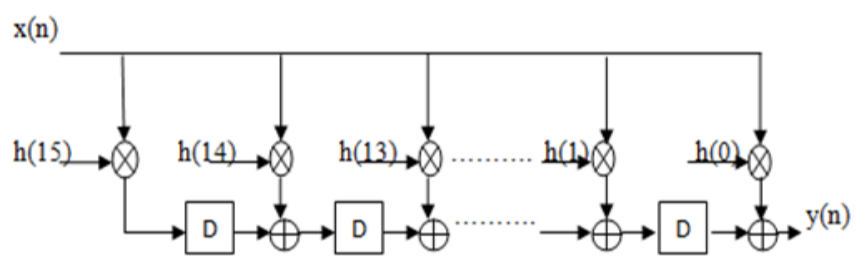

Figure 4. DFG of transpose form structure for output $\mathrm{y}(\mathrm{n})$ of length $\mathrm{n}=16$

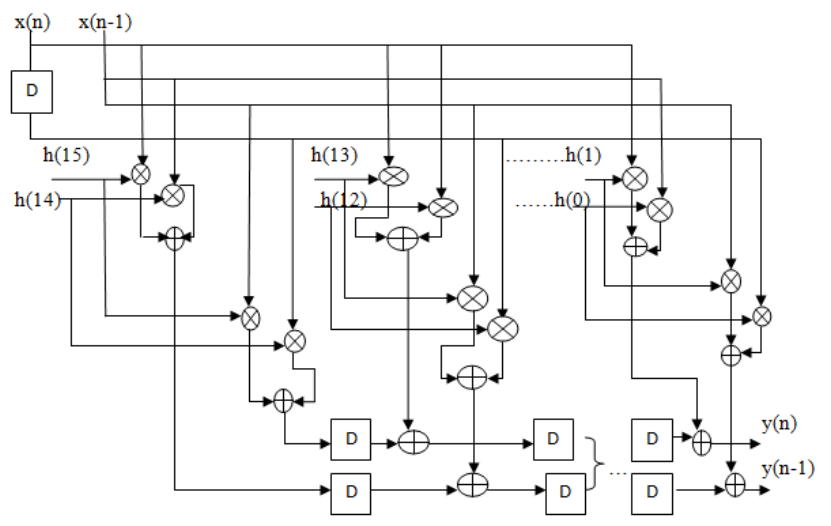

Figure 5. Block Formulation for Transpose Form FIR Filter length $\mathrm{n}=16$

This is the block transpose form configuration of FIR filter with 16 coefficients. The data flow transfer of multipliers of this transpose form configuration of FIR filter is shown in above figure. This is the DFG of block FIR filter of 16 coefficients. It is used to implement proposed structure for reconfigurable applications.

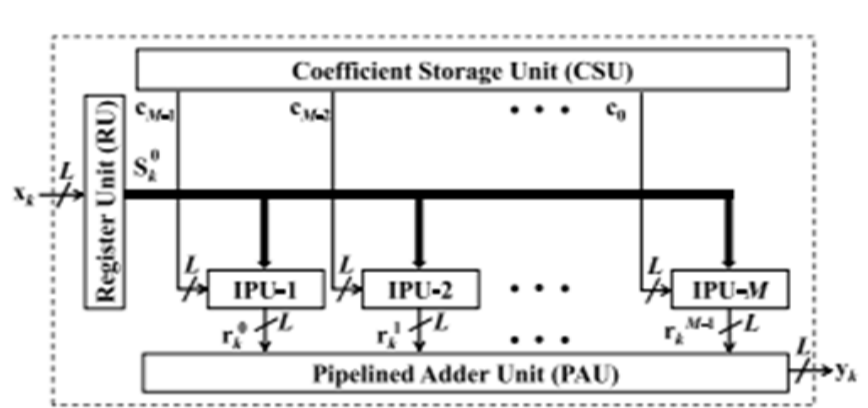

Figure 6. Block transpose of FIR filter for reconfigurable application Simulation results.

\section{WAVEFORMS FOR EXISTING DFG of $\mathrm{N}=4$ :}

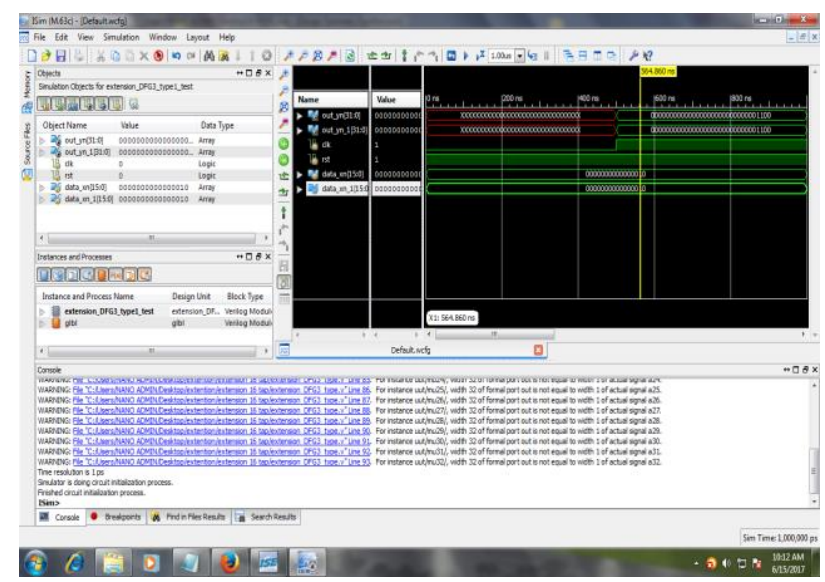

Figure 7. Waveforms for block transpose form configuration for reconfigurable application of $n=6$

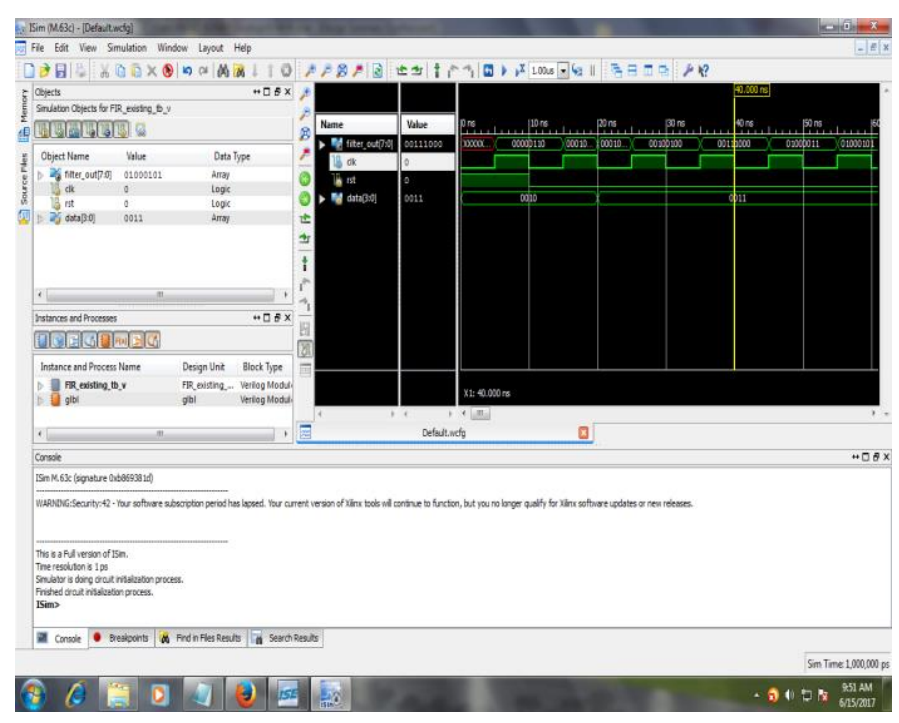

Figure 8.

WAVEFORMS FOR PROPOSED DFG OF N=16: 


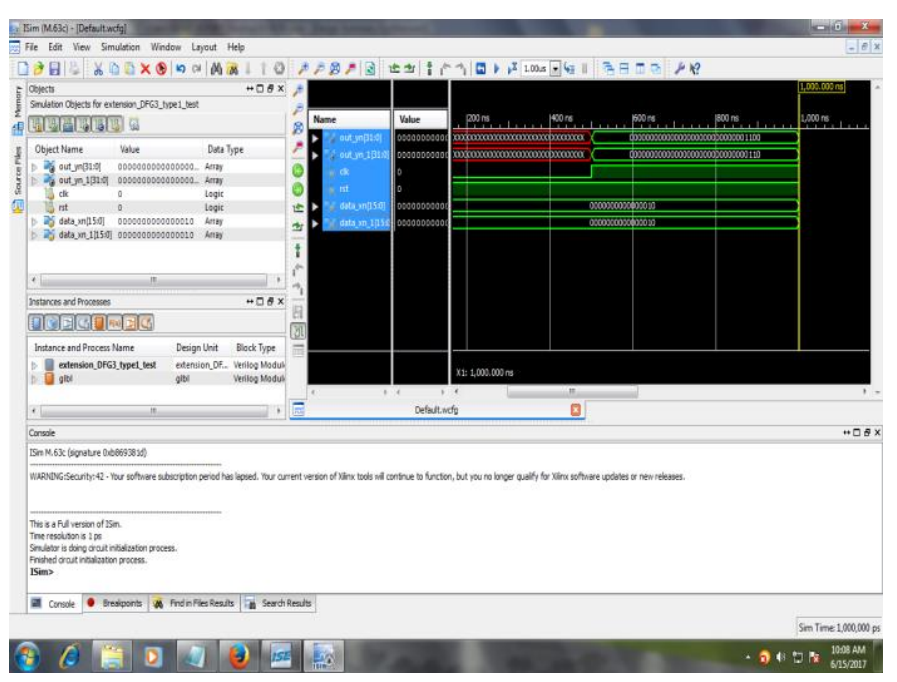

Figure 9. Waveforms for proposed block transpose form configuration for reconfigurable application of

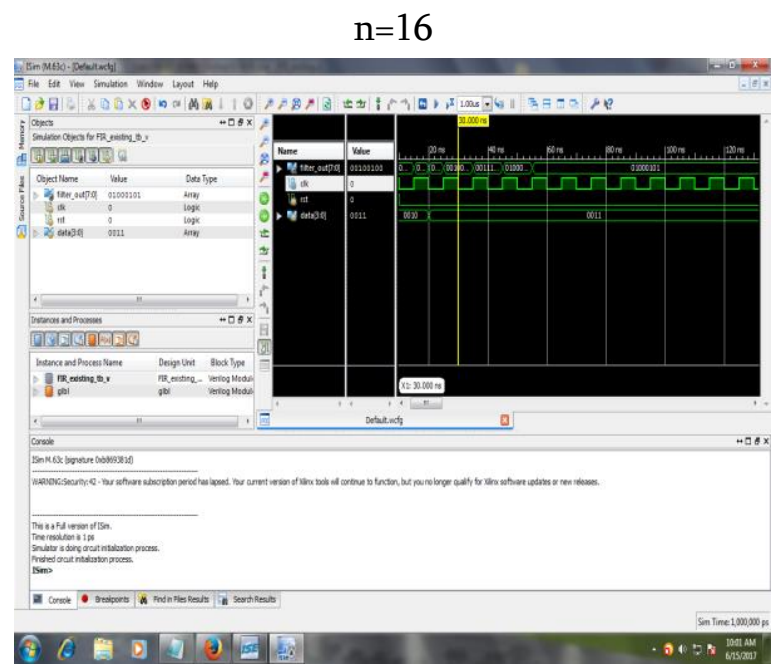

Figure 10.

\section{SYNTHESIS RESULTS}

Table1 : Comparison of existing DFG and proposed DFG

\begin{tabular}{|l|l|l|}
\hline Parameter & $\begin{array}{l}\text { Existing } \\
\text { system }\end{array}$ & $\begin{array}{l}\text { Proposed } \\
\text { system }\end{array}$ \\
\hline No.of 4 input LUTs & 295 & 271 \\
\hline No. of slices & 173 & 149 \\
\hline Time delay & $13.226 \mathrm{~ms}$ & $4.90 \mathrm{~ms}$ \\
\hline
\end{tabular}

Table 2. Comparison of existing and proposed reconfigurable FIR filter

\begin{tabular}{|l|l|l|}
\hline parameter & $\begin{array}{l}\text { Existing } \\
\text { system } \\
\text { of } \\
\text { order(6) }\end{array}$ & $\begin{array}{l}\text { Proposed } \\
\text { system of } \\
\text { order(16) }\end{array}$ \\
\hline No. of 4 input LUTs & 12 & 11 \\
\hline Time delay & $3.724 \mathrm{~ms}$ & $3.724 \mathrm{~ms}$ \\
\hline
\end{tabular}

\section{CONCLUSION}

In this, block transpose form configuration of FIR filter is implemented with a greater number of coefficients. This block FIR filter is used to implement reconfigurable applications.

The delay efficient FIR filter in transpose form configuration is implemented in Xilinx and synthesized report has been generated. The synthesis results show that, the delay of the block transpose form configuration of reconfigurable FIR filter with higher order is similar to that of block transpose configuration of reconfigurable FIR filter with lower order. By using a greater number of coefficients, the performance of the system is increased.

\section{REFERENCES}

[1]. Prof. J. G. Proakis and D. G. Manolakis, Digital Signal Processing: Principles, Algorithms and Applications. Upper Saddle River, NJ, USA:Prentice-Hall, 1996.

[2]. T. Hentschel and G. Fettweis, "Software radio receivers," in CDMA Techniques for Third Generation Mobile Systems. Dordrecht, The Netherlands: Kluwer, 1999, pp. 257-283.

[3]. E. Mirchandani, R. L. Zinser, Jr., and J. B. Evans, "A new adaptive noise cancellation scheme in the presence of crosstalk speech 
signals]," IEEE Trans. Circuits Syst. II, Analog Digit. Signal Process., vol. 39, no. 10, pp. 681694, Oct. 1995.

[4]. D. Xu and J. Chiu, "Design of a high-order FIR digital filtering and variable gain ranging seismic data acquisition system," in Proc. IEEE Southeastcon, Apr. 1993, p. 1-6.

[5]. J. Mitola, Software Radio Architecture: ObjectOriented Approaches to Wireless Systems Engineering. New York, NY, USA: Wiley, 2000.

[6]. A. P. Vinod and E. M. Lai, "Low power and high-speed implementation of FIR filters for software defined radio receivers," IEEE Trans. Wireless Commun., vol. 7, no. 5, pp. 16691675, Jul. 2006.

[7]. J. Park, W. Jeong, H. Mahmoodi-Meimand, Y. Wang, H. Choo, and K. Roy, "Computation sharing programmable FIR filter for low-power and high-performance applications," IEEE J. Solid State Circuits, vol. 39, no. 2, pp. 348-357, Feb. 2004.

[8]. K.-H. Chen and T.-D. Chiueh, "A low-power digit-based reconfigurable FIR filter," IEEE Trans. Circuits Syst. II, Exp. Briefs, vol. 53, no. 8, pp. 617-621, Aug. 2006.

[9]. R. Mahesh and A. P. Vinod, "New reconfigurable architectures for implementing FIR filters with low complexity," IEEE Trans. Comput.-Aided Design Integr. Circuits Syst., vol. 29, no. 2, pp. 275-288, Feb. 2010.

[10]. S. Y. Park and P. K. Meher, "Efficient FPGA and ASIC realizations of a DA-based reconfigurable FIR digital filter," IEEE Trans. Circuits Syst. II, Exp. Briefs, vol. 61, no. 7, pp. 511-515, Jul. 2014.

[11]. P. K. Meher, "Hardware-efficient systolization of DA-based calculation of finite digital convolution," IEEE Trans. Circuits Syst. II, Exp. Briefs, vol. 53, no. 8, pp. 707-711, Aug. 2006.

\section{Cite this article as :}

R. Yerriswamy, Dr. D. Vishnu Vardhan, Sankar Lal Sharma, "A High-Performance FIR filter Architecture for Reconfigurable Applications", International Journal of Scientific Research in Science and Technology (IJSRST), Online ISSN : 2395-602X, Print ISSN : 2395-6011, Volume 6 Issue 1, pp. 420-424, January-February 2019. Available at doi : https://doi.org/10.32628/IJSRST196158 Journal URL : http://ijsrst.com/IJSRST196158 